United Therapeutics. DK is chief medical officer of Eicos Sciences, Inc., Elizabeth Volkmann Consultant of: Boehringer Ingelheim, Grant/research support from: Corbus, Forbius, Boehringer Ingelheim, Øyvind Midtvedt Shareholder of: Son of owner of ACHIM., Henriette Didriksen Speakers bureau: Travel bursary GSK, Alvilde Dhainaut: None declared, Anna-Kristine H Halse: None declared, Gunnstein Bakland: None declared, Inge Christoffer Olsen: None declared, Maiju E Pesonen: None declared, Øyvind Molberg: None declared, Anna-Maria Hoffmann-Vold Consultant of: Actelion, ARXX, Bayer, Boehringer Ingelheim, Medscape, Merck Sharp \& Dohme, Lilly and Roche., Grant/research support from: Actelion, ARXX, Bayer, Boehringer Ingelheim, Medscape, Merck Sharp \& Dohme, Lilly and Roche.

DOI: 10.1136/annrheumdis-2021-eular.2184

\section{AB0434 VITAMIN D IN SYSTEMIC SCLEROSIS PATIENTS}

M. M. Sirufo ${ }^{1}$, F. De Pietro ${ }^{1}$, M. Raggiunti ${ }^{1}$, M. De Martinis ${ }^{1}$, L. Ginaldi ${ }^{1}$. ${ }^{1}$ Department of Life, Health \& Environmental Sciences, University of L'Aquila, Italy; Allergology and Clinical Immunology Unit, AUSL 04, Teramo, Italy., Department of Life, Health \& Environmental Sciences, University of L'Aquila, Italy; Allergology and Clinical Immunology Unit, AUSL 04, Teramo, Italy., Teramo, Italy

Background: Systemic Sclerosis (SSc) is a generalized and systemic autoimmune disease that affects the connective tissue of the skin and internal organs, especially kidneys, heart and lungs [1].

Objectives: Numerous data from recent literature confirm the regulatory action of vitamin D on the immune system and, therefore, how a deficit of this micronutrient can lead to alterations in the immune response, as is known to happen in many allergic and autoimmune diseases [2]. We studied the association between vitamin D levels and SSc, evaluating their correlation with the characteristic manifestations of the pathology.

Methods: We dosed the serum levels of 25 hydroxy-vitamin D in 42 patients with SSc (average age $64.63+/-7.33$ ) and 40 healthy controls comparable for sex and age. The diagnosis of SSc was formulated in accordance to 2013 ACR/EULAR criteria. None of the subjects involved in the study took vitamin D products.

Results: Patients' vitamin D levels $(26.22+/-9.82 \mathrm{ng} / \mathrm{ml})$, although they tended to be lower than controls $(27.80+/-16.53 \mathrm{ng} / \mathrm{ml})$, showed no significant decrease. In patients with pulmonary fibrosis, vitamin $D$ levels were $23.28+/-12.30$ lower than in patients with trophic ulcers and compared to patients without complications $26.07+/-9.92$, although with not statistically significant values. No statistically significant difference was found between vitamin $D$ levels in patients with trophic ulcers compared to controls without complications.

Conclusion: According to the studies in the literature, in our sample, vitamin D deficiency was therefore greater in patients with SSc, especially with pulmonary fibrosis, than in controls [3,4]. Vitamin D levels in diffused-type SSc patients were significantly lower than those in limited-type SSc patients. Further studies are needed to clarify the role that vitamin D deficiency plays in SSc, but lower vitamin $\mathrm{D}$ levels in these patients may suggest the need to monitor blood levels of vitamin $\mathrm{D}$ and supplement it appropriately.

REFERENCES:

[1] De Martinis M, Ciccarelli F, Sirufo MM, Ginaldi L. An overview of environmental risk factors in systemic sclerosis. Expert Rev Clin Immunol. 2016;12(4):465-78. doi: 10.1586/1744666X.2016.1125782. Epub 2015 Dec 19. PMID: 26610037

[2] Yang, CY., Leung, P.S.C., Adamopoulos, I.E. et al. The Implication of Vitamin D and Autoimmunity: a Comprehensive Review. Clinic Rev Allerg Immunol 45, 217-226 (2013). https://doi.org/10.1007/s12016-013-8361-3.

[3] Trombetta AC, SmithV, Gotelli E, Ghio M, Paolino S, Pizzorni C, et al. (2017) Vitamin $D$ deficiency and clinical correlationsin systemic sclerosis patients: A retrospective analysis for possible future developments. PLoS ONE 12(6): e0179062.https://doi.org/10.1371/journal. pone.0179062.

[4] Sarita Gupta, Vikram K. Mahajan, Rajinder S. Yadav1, Karaninder S. Mehta, Satya Bhushan1, et al. Evaluation of Serum Vitamin D Levels in Patients with Systemic Sclerosis and Healthy Controls: Results of a Pilot Study Article July 2018 DOI: 10.4103/idoj.IDOJ_328_17.

Disclosure of Interests: None declared

DOI: 10.1136/annrheumdis-2021-eular.2268

\section{AB0435 CAPILAROSCOPY DOES NOT PREDICT CARDIOVASCULAR EVENTS IN PATIENTS WITH SYSTEMIC SCLEROSIS: A RETROSPECTIVE STUDY}

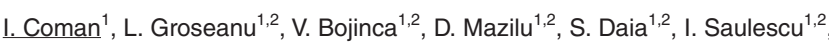
C. L. Constantinescu ${ }^{1,2}$, M. Abobului ${ }^{1,2}$, A. Borangiu ${ }^{1,2}$, M. M. Negru ${ }^{1,2}$, C. Cobilinschi ${ }^{1,2}$, M. Duna ${ }^{1}$, D. Predeteanu ${ }^{1,2}$, F. Berghea ${ }^{1,2}$, A. Balanescu ${ }^{1,2}$, R. Ionescu ${ }^{1,2}$. ${ }^{1}$ Saint Mary Hospital, Rheumatology, Bucuresti, Romania; ${ }^{2}$ Carol Davila University of Medicine and Pharmacy, Medicine, București, Romania
Background: Systemic sclerosis is associated with increased risk of cardiovascular disease (CVD) (1) and studies using MRI suggest that microvascular disease has an important role (2). Capillaroscopy is a non invasive and safe technique that assesses peripheral microvascular damage (3).

Objectives: The objective of this study was to evaluate the predictive value of the capillaroscopy in relation to major adverse cardiovascular events (MACE).

Methods: Retrospective study with three timepoints (at baseline, at 5 and 10 years) including patients with scleroderma from EUSTAR center 096. Data were collected from the registry and observation papers. We performed capillaros copy to all patients at the time of inclusion in the EUSTAR registry (2004) and at baseline (2009). Also, CV risk scores were calculated at baseline and were reassessed at 5 and at 10 years using the SCORE calculator. Risk score was considered low if was between 0 and 2 , moderate 3-9 and high $>10$. The relation between capillaroscopy and MACE was tested in using bivariate regression.

Results: Of 22 patients, mean (standard deviation (SD)) age was 43.1 (11.5), all patients were females, mean (SD) disease duration was 5.4 (4.5). During the 10 years of follow up, 5 (22.7) patients had been lost of follow up and 8 (36.4) patients had died. Only 9 (40.9) patients completed the 10 year follow up. At the time of inclusion in the EUSTAR, one patient showed early scleroderma pattern at capilaroscopy, 15 had active pattern and 6 patients had late pattern Capillaroscopic scoring showed $2(9.1)$ patients with disarranged $(<50 \%)$ aspect, 5 (22.7) patietns with disarranged (>50\%) aspect, 4 (18.2) patients with local paucity, 10 (45.5) patients with enlarged loop bordering local paucity and 1 patient (4.5) with complete paucity.

Capilaroscopy at baseline showed active pattern in 4 patients and late pattern in 18 patients. Thus, 13 (59.1) of patients had a progression of the disease at capilaroscopy before follow up.

At baseline, 14 (63.6) patients had traditional CV risk factors. Cardiovascular risk scores found 21 (95.5) patients with a low risk score and 1 (4.5) patient with a moderate risk score. At 5 years 11 (0.5) patients had a low risk score and 1 (4.5) patient had moderate risk score and at 10 years, 5 (22.7) and 2 (9) patients had low and moderate risk scores, respectively. We found 6.2 MACE/100 patient-yea and 5.5 deaths/100 patient-year.

Capillaroscopy $(p=0.684)$, disease progression on capillaroscopy $(p=0.781)$, capillaroscopic scoring $(p=0.92)$ and CV risk score $(p=0.98)$ were not predictive factors of MACE.

Conclusion: Patients with systemic sclerosis are at high risk of MACE and traditional CV risk scores underestimate this risk. Changes/progression on capilaroscopy is not predictive for MACE. However, this hypothesis needs to be tested on a bigger cohort.

\section{REFERENCES:}

[1] Xintao Cen, SIning Feng, Shanshan Wei, Lu Wan, Ledong Sun, Systemic sclerosis and risk of cardiovascular disease: A PRISMA - compliant systemic review and meta-analysis of cohort studies, Medicine (Baltimore), 2020, 99(47)

[2] N Galea, E Rosato, A Gigante, C Borrazzo, A Fiorelli et al, Early myocardial damage and microvascular dysfunction in asymptomativ patients with systemic sclerosis: A cardiovascular magnetic resonance study with cold pressor test, PLoS One 2020, 15 (12)

[3] F Ingegnoli, R Gualtierotti, A systematic overview on the use and relevance of capillaroscopy in systemic sclerosis, Expert Rev CLin Ummunol, 2013, 9(11):1091-7

Disclosure of Interests: None declared

DOI: 10.1136/annrheumdis-2021-eular.2430

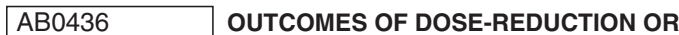 DISCONTINUATION OF TOCILIZUMAB IN PATIENTS WITH EARLY DIFFUSE CUTANEOUS SYSTEMIC SCLEROSIS}

Y. Isomura ${ }^{1}$, Y. Yamasaki ${ }^{1}$, Y. Shirai ${ }^{1}$, M. Kuwana ${ }^{1} .{ }^{1}$ Nippon Medical School, Graduate School of Medicine, Department of Allergy and Rheumatology, Tokyo, Japan

Background: Potential efficacy and favorable safety profiles of tocilizumab (TCZ) have been demonstrated in patients with diffuse cutaneous systemic sclerosis (dcSSc) [1, 2]. However, clinical outcomes after dose-reduction or discontinuation of TCZ due to an improvement of skin thickness remain unclear.

Objectives: To investigate the clinical outcomes after dose-reduction or discontinuation of TCZ in patients with dcSSc in a real-world setting.

Methods: This is a single-center, retrospective, observational study using a database of consecutive SSc patients who visited our center between April 2014 and October 2020. For this study, we selected eligible patients from the database based on the following criteria: patients who (i) fulfilled the ACR/EULAR classification criteria, (ii) were classified as having dcSSc, (iii) had been treated with TCZ for at least 6 months, and (iv) were follow-up $>6$ months after TCZ introduction. Clinical information including demographic and clinical characteristics at TCZ introduction; dosing, administration route, and adherence of TCZ; and serial clinical parameters 